\title{
Relationship between Endogenous Polyamines and Floral Bud Differentiation in Chrysanthemum morifolium under Short-day Conditions
}

\author{
Jun-e Guo ${ }^{1,2}$, Tian $\mathrm{Li}^{1,2}$, Xianzhi Sun ${ }^{1,2^{*}}$, Chengshu Zheng ${ }^{1,2}$, and Xia Sun ${ }^{1,2}$ \\ ${ }^{1}$ College of Horticulture Science and Engineering, Shandong Agricultural University, Tai' an 271018, PR China \\ ${ }^{2}$ State Key Laboratory of Crop Biology, Shandong Agricultural University, Tai' an 271018, PR China
}

\begin{abstract}
This study was carried out to investigate the relationship between endogenous polyamines (PAs) and floral bud differentiation in chrysanthemum (Chrysanthemum morifolium). In this study, PA content (free, bound, and conjugated) in apical buds, leaves, and roots changed appreciably during floral bud differentiation. PAs accumulated during series of processes such as floral induction, differentiation of floret primordia, and crown formation in apical buds; changes in PAs in apical buds may have a relationship with those in leaves and roots. The levels of free PAs and conjugated PAs [putrescine (Put) and spermine $(\mathrm{Spm})]$ in apical buds rapidly increased during the initiation stage of floral bud differentiation, while free and conjugated spermidine (Spd) reached their highest levels at the stage of floret primordium differentiation. In the free, conjugated, and bound PA fractions, the changes in Spm content were negligible compared to those of Put and Spd throughout the experiment. These findings indicate that PAs participate in regulating the process of flower bud differentiation in chrysanthemum.
\end{abstract}

Additional key words: floral initiation, microscopic observation, photoperiod induction, polyamine metabolism, HPLC

\section{Introduction}

Polyamines (PAs) with low molecular weight, are polycationic and nitrogenous growth regulators that exist in all living cells. The most common free PAs in plants are diamine putrescine (Put), triamine spermidine (Spd), and tetraamine spermine (Spm), with rare accumulation of longchain PAs under special conditions (Buchanan et al., 2000; Martin-Tanguy, 2001). They exist in three forms in the cell: as free cations (free PAs), covalently bound to lowmolecular-weight phenolic compounds such as hydroxycinnamic acids (conjugated PAs), and bound to macromolecules or membranes (bound PAs) (Rajam et al., 2004). It has been demonstrated that polyamines were translocated only in the free form (Antognoni et al., 1998). PAs are essential for cell growth and participate in numerous physiological processes (Igarashi and Kashiwagi, 2010). These compounds are fully protonated under physiological conditions and can react as polyvalent cations with numerous cell constituents, such as nucleic acids, ATP, specific proteins, and phospholipids. PAs play important roles in plants, such as promoting plant growth, inhibiting the activity of protease and RNA enzymes and increasing plant resistance and so on. And they also play important roles in floral bud differentiation (Aribaud and Martin-Tanguy, 1994) and pollen formation (Wimalasekera et al., 2011) in horticultural crops.

Plant flower bud differentiation is an important and complex process affected by many internal and external factors. The importance of polyamines in flower bud differentiation has been confirmed by numerous laboratory studies (Aribaud and Martin-Tanguy, 1994; Huang et al., 2004; Meijón et al., 2011; Wimalasekera et al., 2011). Endogenous PA concentrations in plants change dramatically during the transition from vegetative phase to reproductive (Applewhite et al., 2000; Aribaud and Martin-Tanguy, 1994; Davis, 2009; Meijón et al., 2011; Wimalasekera et al., 2011).

\footnotetext{
*Corresponding author: sunxianzhi@126.com

※ Received 10 March 2014; Revised 17 September 2014; Accepted 23 September 2014. This work was financially supported by National Natural Science Foundation of China (No. 31101564).

(C) 2015 Korean Society for Horticultural Science
} 
Conjugated PAs have been shown to play a key role in floral development (Martin-Tanguy, 1997) and be of particular significance for regulation of PA concentration (Bagni and Tassoni, 2001).

Chrysanthemum is one of the best-known flowers worldwide. The floral bud differentiation of chrysanthemum associated with endogenous hormones (Feng and Yang, 2011), endogenous PAs (Aribaud and Martin-Tanguy, 1994), environmental factors (Yang et al., 2006), carbohydrates (Lang et al., 2008) and so on. Regarding the role of polyamines in flowering of chrysanthemum, Aribaud and Martin-Tanguy (1994) described changes in PAs in apical buds and proposed that some polyamines may be involved in regulating floral initiation. Since that report, the function of PAs metabolism in chrysanthemum remained an outstanding question.

Chrysanthemum morifolium 'Jinba', a short-day plant, was used as the study material, to study the dynamic changes of endogenous PAs of chrysanthemum during floral bud differentiation process, attempted to learn the effects of the metabolic mechanism of PAs to the floral bud differentiation and to identify the relationship between flower bud differentiation periods and endogenous PAs.

\section{Materials and Methods}

\section{Plant Growth Conditions and Sampling}

The experiment was carried out in a greenhouse at the
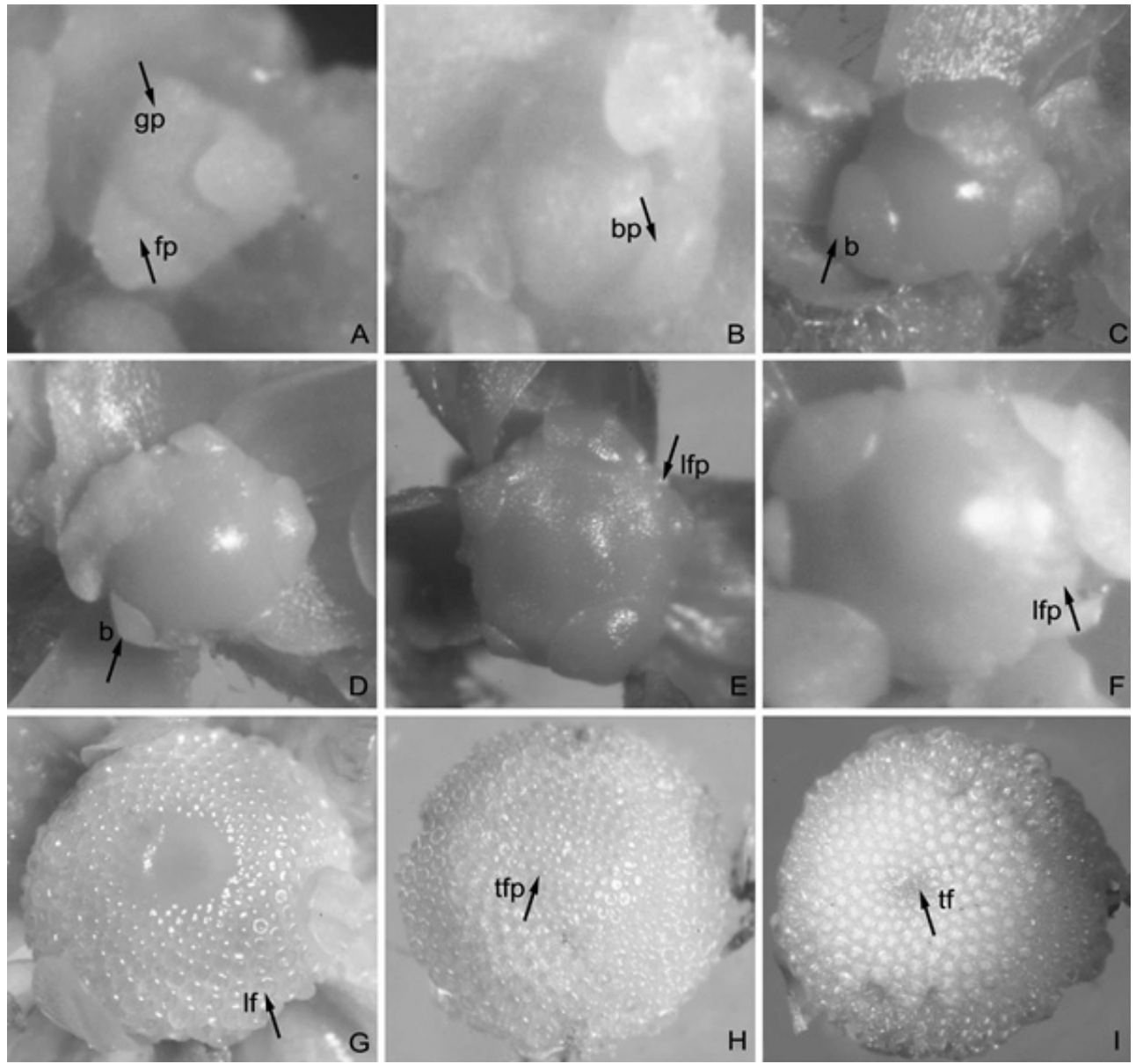

Fig. 1. The stages of the floral bud differentiation of Chrysanthemum morifolium 'Jinba'. A: The vegetative stage; B: The initiative stage of floral bud differentiation; C: The initial stage of involucre primordia differentiation; D: The final stage of involucre primordia differentiation; E: The initial stage of floret primordia differentiation; F: The final stage of floret primordia differentiation; G: The initial stage of crown formation; H: The metaphase stage of crown formation; I: The final stage of crown formation. Abbreviations: fp: Foliage primordia; gp: Growing point; bp: Bract primordia; b: Bract; lfp: Ligulate flower primordia; If :Ligulate flower; tfp: Tubular flower primordia; tf: Tubular flower. The figure size: $17 \mathrm{~cm}$. Magnification: 28-30 times. 
Shandong Agriculture University, China, from 6 April to 14 June 2012. Chrysanthemum morifolium 'Jinba', a short-day plant, was used as the study material. The temperature of the greenhouse ranged from approximately 18 to 30 .

Rooted cuttings of chrsyantehmum, 'Jinba' were grown under long-day conditions in the greenhouse. Short-day treatment (SD, $10 \mathrm{~h}$ light/14 h dark) began on 15 May 2012 (6:00 PM) when plants were approximately $55 \mathrm{~cm}$ tall. Day length was shortened by shading from 6:00 PM to 8:00 AM the following day. The plant materials analysed in this experiment were collected on the day of the photoperiodic treatment (15 May, 8:00 AM), and then one sample was collected (with 3 replicates) every 3 days until 14 June. The sample materials collected included apical buds (with two lobes that had not fully expanded and were approximately $1.5 \mathrm{~cm}$ long), functional leaves (the 3rd and 4th fully expanded mature leaves from top to bottom), and roots (root tips, about $1.0 \mathrm{~cm}$ long) of 10-20 plants. After determination of fresh mass, the samples were frozen in liquid nitrogen and stored at $-80^{\circ} \mathrm{C}$ for analysis of PAs and oxidase activity. PA titres and oxidase activities were taken for analysis at 5 major stages as follows: (1) the vegetative stage; (2) the initiative stage of floral bud differentiation; (3) the stage of involucre primordia differentiation; (4) the stage of floret primordia differentiation; and (5) the stage of crown formation.

\section{Observation of Flower Bud Differentiation}

From the first day of shading, 10 apical buds were collected every three days and preserved in FAA stationary liquid. We observed and recorded the flower bud differentiation process which was subdivided into 9 stages as described below with a stereoscopic microscope (OLYMPUS, SZX10-1131; Japan) (Fig. 1 and Table 1).

\section{Extraction and Measurement of PAs}

We monitored the production of three PAs, putrescine (Put), spermidine (Spd), and spermine (Spm) (Fig. 2). Benzoyl chloride and PA standards were obtained from Sigma-Aldrich (Shanghai, China; imported from USA).

Free, conjugated, and bound PAs were extracted and analysed by the method described by Zhou and Yu (2010).

\section{Statistical Analysis}

All data were analysed and presented as means SE of three replicates using SPSS software (ver. 17.0, Chicago, USA), and differences among means were determined by Duncan's multiple test.

Table 1. The bud differentiation of every period of Chrysanthemum morifolium 'Jinba'.

\begin{tabular}{|c|c|c|c|c|c|c|c|c|c|}
\hline \multirow{2}{*}{ Sampling time } & \multicolumn{9}{|c|}{ Numbers of apical buds reached at each differentiation stage } \\
\hline & I & II & III & IV & $\mathrm{V}$ & VI & VII & VIII & IX \\
\hline May 15 & 10 & & & & & & & & \\
\hline May 18 & 6 & 4 & & & & & & & \\
\hline May 21 & 1 & 5 & 4 & & & & & & \\
\hline May 24 & & 1 & 8 & 1 & & & & & \\
\hline May 27 & & & 2 & 8 & & & & & \\
\hline May 30 & & & & 1 & 6 & 3 & & & \\
\hline June 2 & & & & & 1 & 6 & 3 & & \\
\hline June 5 & & & & & & 2 & 7 & 1 & \\
\hline June 8 & & & & & & & 2 & 6 & 2 \\
\hline June 11 & & & & & & & & 3 & 7 \\
\hline June 14 & & & & & & & & 1 & 9 \\
\hline
\end{tabular}

I - IX: I: The vegetative stage; II: The initiative stage of floral bud differentiation; III: The initial stage of involucre primordia differentiation; IV: The final stage of involucre primordia differentiation; V: The initial stage of floret primordia differentiation; VI: The final stage of floret primordia differentiation; VII: The initial stage of crown formation; VIII: The metaphase stage of crown formation; IX: The final stage of crown formation. From the first day of shading, 10 apical buds were collected every three days to observed and recorded the flower bud differentiation process. The number in table mean that how many apical buds at this stage on that day. For example, the number 10 in black are all at stage I on May 15. Blanks in the column mean that no apical buds were found in the stage on that day. 


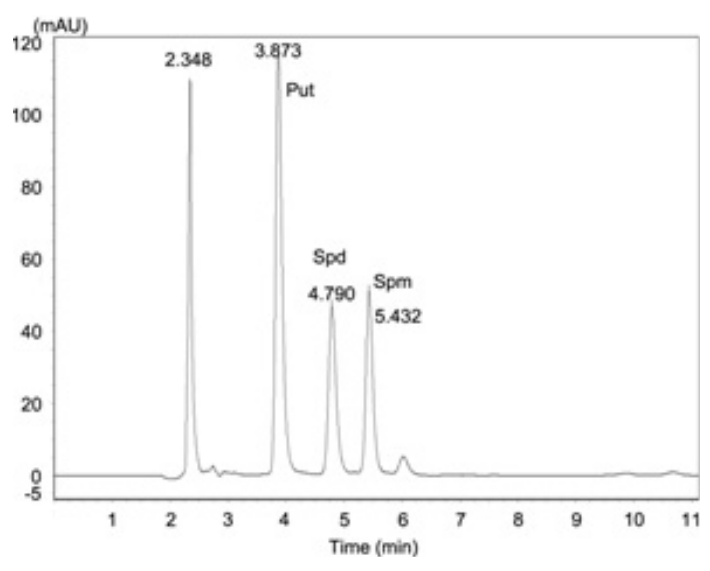

Fig. 2. Separation of standard mixture of biogenic amines [putrescine (Put), spermidine (Spd) and spermine (Spm)] as benzoyl chloride. Concentration of the amines was 25 $\mathrm{mg} \cdot \mathrm{kg}^{-1}$ in the acetonitrile solution for injection.

\section{Results}

\section{Changes in PA Levels in Apical Bud}

Content of free, conjugated, and bound PAs changed appreciably during floral bud differentiation (Fig. 3). The highest free and conjugated Put and free Spm content were observed during stage 2, while free and conjugated Spd reached their highest levels at stage 4 . Free Spd was the predominant PA at stages 4 and 5, representing 69-70\% and $86-88 \%$, respectively, of the free-PA pool. Spm was not detected at the stage 1 . Content of bound Spd were much higher than those of bound Put and Spm throughout the process of floral bud differentiation despite a steady decrease as differentiation progressed. Bound Put and Spm changed more or less in parallel, in contrast to the trend in free Spd. Free Put reached its lowest level at stage 5; content of free Put, Spd, and Spm were higher during the entire period of floral bud differentiation (stage 5) than during the vegetative stage (stage 1).

During floral bud differentiation, content of free Spd and free and conjugated total PAs changed in parallel, and respectively reached their highest levels at the stage 2 and stage 4 , as well as, content of the conjugated total PAs higher than free PAs (Fig. 3).

\section{Changes in PA Levels in Leaves}

The highest concentration of free Put in leaves was recorded during stage 1 (Fig. 4). Free Spm and Spd concentrations were highest at stage 4 and the concentration of Spd was significantly higher than that of Put or Spm

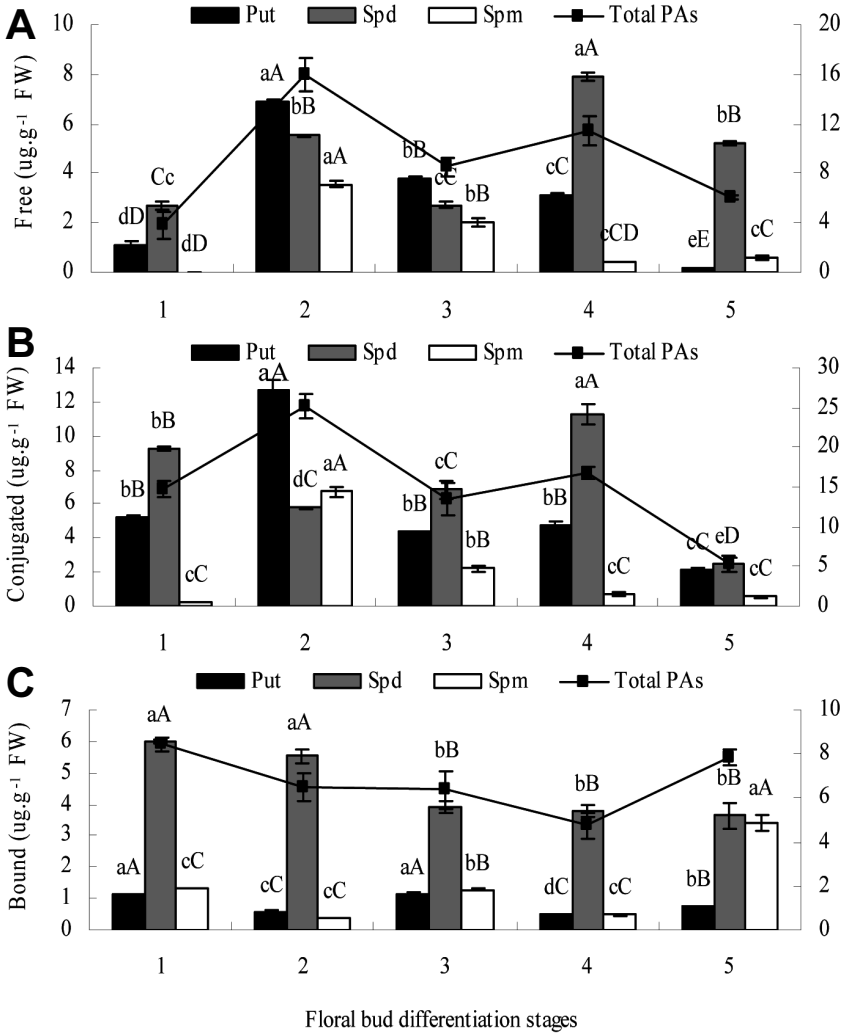

Fig. 3. Changes in the concentration of free $(A)$, conjugated (B) and bound (C) polyamines [putrescine (Put), spermidine (Spd) and spermine (Spm)], and total PAs in apical buds of Chrysanthemum morifolium 'Jinba' during different floral bud differentiation stages. The number 1-5 means the stages of floral bud differentiation: Stage 1: The vegetative stage; Stage 2: The initiative stage of floral bud differentiation; Stage 3: The stage of involucre primordia differentiation; Stage 4: The stage of floret primordia differentiation; Stage 5: The stage of crown formation. The same as below. Withingraph means followed by the same small and capital letters are not significantly different by Duncan's multiple range test at $p<0.05$ and $p<0.01$, respectively. Vertical bars represent mean $\pm \mathrm{SE}(\mathrm{n}=3)$.

at this stage. During all of the other stages, concentrations of free Put were higher than those of free Spd and Spm. Conjugated Put predominated during the entire period of floral bud differentiation. The concentrations of bound Spd were significantly higher than those of Put and Spm from the initiative stage until the end of floral bud differentiation (Fig. 4).

Free and conjugated total PAs decreased at stage 2, and the content of free and conjugated total PAs lower than the vegetative stage during floral bud differentiation. 

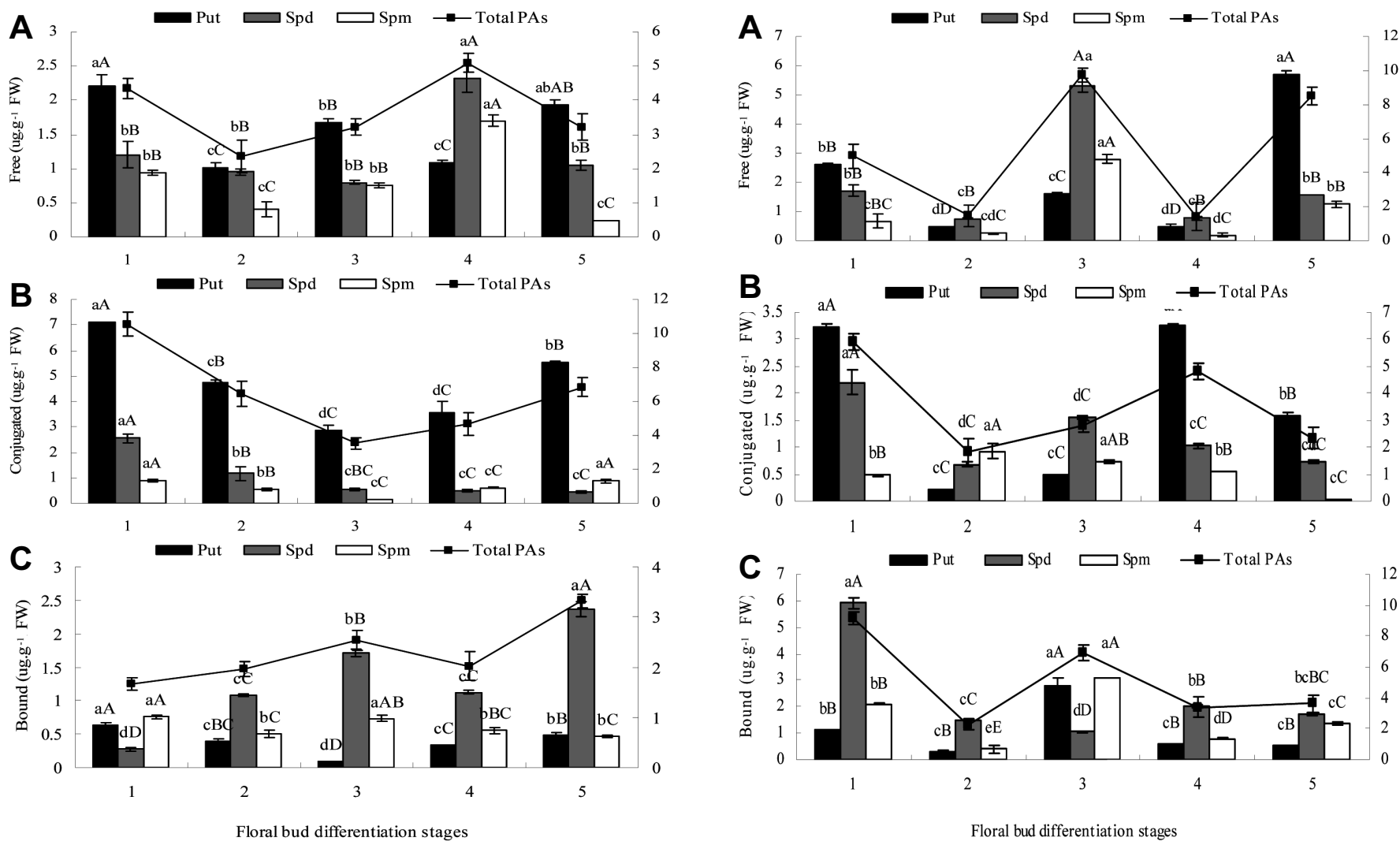

Fig. 4. Changes in the concentration of free (A), conjugated (B) and bound (C) polyamines [putrescine (Put), spermidine (Spd) and spermine (Spm)], and total PAs in leaves of Chrysanthemum morifolium 'Jinba' during different floral bud differentiation stages. Within-graph means followed by the same small and capital letters are not significantly different by Duncan's multiple range test at $p<0.05$ and $p<0.01$, respectively. Vertical bars represent mean $\pm \operatorname{SE}(n=3)$.

\section{Changes in PA Levels in Roots}

In roots, free Put, Spd, and Spm changed more or less in parallel during the entire period of flower bud differentiation, showing an initial decrease at the initiative stage and reaching a peak at differentiation of involucre primordia (Fig. 5). A decrease was again observed until differentiation of floret primordia, followed by an increase at stage 5 . After short-day treatment, the content of the conjugated Put and Spd rapidly decreased during the initiative stage. Bound Spd concentrations were significantly lower during floral bud differentiation $(<2.1)$ than during the vegetative stage (> 5.9) (Fig. 5).

Throughout the whole experiment, free and bound total PAs changed in parallel, and had the significantly lower content at stage 2 and stage 4 (Fig. 5).

Fig. 5. Changes in the concentration of free (A), conjugated (B) and bound (C) polyamines [putrescine (Put), spermidine (Spd) and spermine (Spm)], and total PAs in roots of Chrysanthemum morifolium 'Jinba' during different floral bud differentiation stages. Within-graph means followed by the same small and capital letters are not significantly different by Duncan's multiple range test at $p<0.05$ and $p<0.01$, respectively. Vertical bars represent mean $\pm \operatorname{SE}(n=3)$.

The Changes in Ratio of Put/Spd and Put/(Spd + Spm) during Floral Bud Differentiation

During physiological differentiation, free and conjugated Put/Spd and Put/(Spd + Spm) changed in parallel first increased and then decreased, while conjugated and bound Put/Spd and Put/(Spd + Spm) changed in parallel during morphological differentiation (Table 2). Free and bound Put/Spd and Put/(Spd + Spm) reached their highest levels at stage 3, and conjugated Put/Spd and Put/(Spd + Spm) reached their highest levels at stage 2 (Table 2).

\section{Discussion}

Metabolism of Endogenous PAs in Chrysanthemum during Flower Bud Differentiation

Polyamine regulation of floral bud induction, initiation, 
Table 2. Changes of Put/Spd and Put/(Spd + Spm) of chrysanthemum during floral bud differentiation

\begin{tabular}{lccccccc}
\hline Floral bud & \multicolumn{3}{c}{ Put/Spd } & & \multicolumn{3}{c}{ Put/(Spd + Spm) } \\
\cline { 2 - 5 } \cline { 6 - 8 } Differentiation stages & Free & Conjugated & Bound & & Free & Conjugated & Bound \\
\hline 1 & $0.41 \mathrm{cC}$ & $0.56 \mathrm{cdC}$ & $0.19 \mathrm{bBC}$ & & $0.41 \mathrm{bB}$ & $0.55 \mathrm{cC}$ & $0.15 \mathrm{bB}$ \\
2 & $1.25 \mathrm{bB}$ & $2.20 \mathrm{aA}$ & $0.11 \mathrm{cD}$ & & $0.76 \mathrm{aA}$ & $1.01 \mathrm{aA}$ & $0.10 \mathrm{cC}$ \\
3 & $1.38 \mathrm{aA}$ & $0.65 \mathrm{cBC}$ & $0.30 \mathrm{aA}$ & & $0.79 \mathrm{aA}$ & $0.49 \mathrm{cCD}$ & $0.22 \mathrm{aA}$ \\
4 & $0.40 \mathrm{cC}$ & $0.43 \mathrm{dC}$ & $0.13 \mathrm{cCD}$ & & $0.38 \mathrm{bB}$ & $0.40 \mathrm{dD}$ & $0.11 \mathrm{cBC}$ \\
5 & $0.04 \mathrm{dD}$ & $0.86 \mathrm{bB}$ & $0.21 \mathrm{bB}$ & & $0.03 \mathrm{cC}$ & $0.70 \mathrm{bB}$ & $0.11 \mathrm{cBC}$ \\
\hline
\end{tabular}

Within-graph means followed by the same small and capital letters are not significantly different by Duncan's multiple range test at $p<0.05$ and $p<0.01$, respectively. The number 1-5 means the stages of floral bud differentiation: Stage 1: The vegetative stage; Stage 2: The initiative stage of floral bud differentiation; Stage 3: The stage of involucre primordia differentiation; Stage 4: The stage of floret primordia differentiation; Stage 5: The stage of crown formation.

transition, and development has been assessed by various researchers. Some authors suggest that PAs could be components of floral stimulus (Caffaro et al., 1994; Havelange et al., 1996), while others maintain that PAs influence transport of a floral stimulus (Applewhite et al., 2000).

In this study, the rapid increase in the free-Put pool in apical buds during stage 2 illustrated its importance to initiation of floral bud formation. This result was contrary to the findings of Aribaud and Martin-Tanguy (1994), possibly because of differences in the study species, temperature, or other factors. However, the content of Put remained low (even lower than that at stage 1) from the differentiation of the involucre primordia until crown formation. Therefore, the role of Put was to promote induction or initiation of the bud, and that low content of Put were beneficial for floral bud morphodifferentiation. Sood and Nagar (2004) reported that, high free Put and Spd concentrations were associated with early stages of flower development, and a steady increase in conjugated Put, Spd and Spm was observed during entire period of flower development, which was different with our results, possibly because of differences in the study species, temperature, or other factors. Tarenghi and Martin-Tanguy (1995) reported that a-difluoromethylornithine (DFMO), an inhibitor of Put synthesis, decreased PA contents in strawberry and inhibited flowering, which could be restored by exogenous application of Put.

PAs might be transferred from leaves to floral buds and inflorescences during the early stages of flower development (Caffaro and Vicente, 1995). Our results showed that, the content of free and conjugated total PAs in apical buds during stage 2 increased rapidly and reached their highest levels, significantly higher than those of the other stages; while free and conjugated total PAs in leaves and three fractions total PAs in roots were decreased. We speculate that, after short-day treatment, conjugated and bound PAs transformed to free PAs in leaves and transport to apical bud, causing abundant PAs accumulate in apical buds where it promoted floral bud differentiation. Studies on chrysanthemum (Aribaud and Martin-Tanguy, 1994), soybean (Caffaro et al., 1994), and tobacco (Cabanne et al., 1981) pointed to the possibility of transport of PAs from young developing leaves to flower buds at the early flowering stages.

According to the findings discussed above, we considered that increased contents of free PAs and conjugated PAs (Put and Spm) were involved in regulating flower induction in C. morifolium 'Jinba', whereas bound Spd, which was the highest in the vegetative phase, was more important for vegetative development.

During differentiation of floret primordia, free and conjugated Spd in apical buds and free Spd in leaves reached its highest level, the total PAs increased, indicated that high content of free and conjugated Spd were might advantageous to initiation and formation of floret primordia. There may be a process of Put and Spm transformed to Spd at this stage, what causing Spd accumulate in apical buds.

Different with our results, Tarenghi and Martin-Tanguy (1995) reported that conjugated Spd was the predominant amide during the earliest stages of floral differentiation in strawberry. Spd has been noted as a marker for flower induction in many plants (Fiala et al., 1988; Huang et al., 2004). Spd- and Spm-deficient Arabidopsis mutants showed early proliferative arrest of apical inflorescence meristems (Hanzawa et al., 2000). In contrasts, Caffaro and Vicente (1995) confirmed that Spd was not involved in flowering induction in Glycine max. Wada et al. (1994) suggested that application of exogenous Spd did not promote flower bud differentiation of Pharbitis nil 'Kidachi'. Studies on Arabidopsis thaliana revealed that the addition of Spd 
promoted flowering when conditions were suboptimal (e.g., SD), but inhibited flowering under more optimal conditions (Applewhite et al., 2000); thus there may be an optimum level of PAs with respect to flowering. Thus, flowering induction by PA appears to vary among species.

During floral bud differentiation, conjugated PAs accumulated and became the predominant form until crown formation, consistent with the findings of Aribaud and Martin-Tanguy (1994). The important role of conjugated PAs during floral bud differentiation has been assessed by researchers, for example, high content of conjugated PAs were associated with the onset of floral bud differentiation and floret primordia formation (Dumas et al., 1981); in a study of azaleas, the vegetative bud contained mostly free PAs while floral buds showed higher content of conjugated forms (Meijón et al., 2009); furthermore, the appearance of conjugated PAs, previously shown to be markers of flowering (Martin-Tanguy, 1985), the same with our experimental results.

\section{The Changes in Ratio of Put/Spd and Put/(Spd + Spm) during Floral Bud Differentiation}

In our study, the higher ratio of free and conjugated Put/Spd and Put/(Spd + Spm) was beneficial for the induction or initiation of the bud, while the higher ratio of free and bound Put/Spd and Put/(Spd + Spm) was advantageous to initiation and formation of floret primordia. A high ratio of Put/Spd has been proposed to indicate active cell division, whereas the decrease in the ratio indicated cell enlargement (Bachrach, 1973).

In conclusion, high content of free and conjugated PAs were advantageous to the onset of floral bud differentiation in chrysanthemum, and that high content of free and

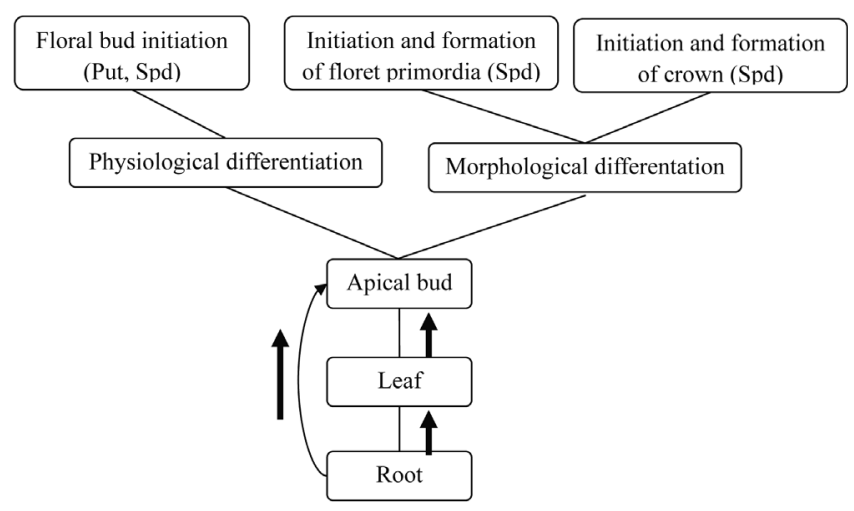

Fig. 6. Metabolism of endogenous polyamines [putrescine (Put) and spermidine (Spd)] in Chrysanthemum morifolium 'Jinba' during flower bud differentiation. conjugated Spd were advantageous to initiation and formation of floret primordia. After short-day treatment, PAs in leaves and roots might transport to apical bud, causing abundant PAs ccumulate in apical buds where it promoted floral bud differentiation (Fig. 6). During floral bud differentiation, conjugated PAs play important role. There are some relationships between floral bud differentiation and the ratio of Put/Spd and Put/(Spd+Spm).

\section{Literature Cited}

Antognoni, F., S. Fornalè, C. Grimmer, E. Komor, and N. Bagni. 1998. Long-distance translocation of polyamines in phloem and xylem of Ricinus communis L. plants. Planta 204:520-527.

Applewhite, P.B., R. Kaur-Sawhney, and A.W. Galston. 2000. A role for spermidine in the bolting and flowering of Arabidopsis. Physiol. Plant. 108:314-320.

Aribaud, M. and J. Martin-Tanguy. 1994. Polyamine metabolism, floral initiation and floral development in chrysanthemum (Chrysanthemum morifolium Ramat.). Plant Growth Regul. 15:23-31

Bachrach, U. 1973. Function of naturally occurring polyamines. Academic Press, New York, USA.

Bagni, N. and A. Tassoni. 2001. Biosynthesis, oxidation and conjugation of aliphatic polyamines in higher plants. Amino Acids. 20:301-317.

Buchanan, B.B., W. Gruissem, and R.L. Jones. 2000. Biochemistry and molecular biology in plants. American Society of Plant Physiologists. Maryland, USA. p. 1-90.

Cabanne, F., M.A. Dalebroux, J. Martin-Tanguy, and C. Martin. 1981. Hydroxycinnamic acid amides and ripening to flower of Nicotiana tabaccum var. xanthi n.c. Physiol. Plant. 53:399-404.

Caffaro, S.V., F. Antognoni, S. Scaramagli, and N. Bagni. 1994. Polyamine translocation following photoperiodic flowering induction in soybean. Physiol. Plant. 91:251-256.

Caffaro, S.V. and C. Vicente. 1995. Early changes in the content of leaf polyamines during the photoperiodic flowering induction in soybean. J. Plant Physiol. 145:756-758.

Davis, S.J. 2009. Integrating hormones into the floral-transition pathway of Arabidopsis thaliana. Plant Cell Environ. 32:1201-1210.

Dumas, E., E. Perdrizet, and J.C. Vallee. 1981. Quantitative evolution of free amino acids and amines in the development of various species of Nicotiana. Physiol. Veg. 19:155-165.

Feng, F. and J.S. Yang. 2011. Relationship between floral bud differentiation and endogenous hormones in autumn-cutting Chrysanthemum morifolium 'Jinba'. Scientia Agr. Sinica. 3:552-561.

Fiala, V., M. Lenard, Y. Querou, and E. Jolivet. 1988. Spermidine as molecular marker of floral induction in the Iris hollandica bulb. C.R. Acad. Sci. Paris. 306:579-582.

Hanzawa, Y., T. Takahashi, A.J. Michael, D. Burtin, D. Long, M. Pineiro, G. Goupland, and Y. Komeda. 2000. ACAULIS5, an Arabidopsis gene required for stem elongation, encodes a 
spermine synthase. EMBO J. 19:4248-4256.

Havelange, A., P. Lejeune, G. Bernier, R. Kaur-Sawhney, and A.W. Galston. 1996. Purtrescine export from leaves in relation to floral transition in Sinapis alba. Physiol. Plant. 96:59-65.

Huang, C.K., B.S. Chang, K.C. Wang, S.J. Her, T.W. Chen, Y.A. Chen, C.L. Cho, L.J. Liao, K.L. Huang, W.S. Chen, and Z.H. Liu. 2004. Changes in polyamine pattern are involved in floral initiation and development in Polianthes tuberosa. J. Plant Physiol. 161:709-713.

Igarashi, K. and K. Kashiwagi, 2010. Modulation of cellular function by polyamines. Intl. J. Biochem. Cell. B. 42:39-51.

Lang, F., C.S. Zheng, C.H. Zhang, and Q.C. Sun. 2008. Change of carbohydrate content in buds and leave of chrysanthemum during floral differentiation. Shandong Agr. Sci. 1:40-42.

Martin-Tanguy, J. 1985. The occurrence and possible functions of hydroxycinnamoyl acid amides in plant. Plant Growth Regul. 3:381-399.

Martin-Tanguy, J. 1997. Conjugated polyamines and reproductive development: Biochemical, molecular and physiological approaches. Physiol. Plant. 100:675-688.

Martin-Tanguy, J. 2001. Metabolism and function of polyamines in plants: recent development (new approaches). Plant Growth Regul. 34:135-148.

Meijón, M., R. Rodríguez, M.J. Cañal, and I. Feito. 2009. Improvement of compactness and floral quality in azalea by means of application of plant growth regulators. Sci. Hortic. 119:169-176.

Meijón, M., M.J. Cañal, H. Fernández, A. Rodríguez, B. Fernández,
R. Rodríguez, and I. Feito. 2011. Hormonal profile in vegetative and floral buds of azalea: levels of polyamines, gibberellins, and cytokinins. J. Plant Growth Regul. 30:74-82.

Rajam, M.V., R. Kumria, and S. Singh. 2004. Molecular biology and genetic engineering of polyamines in plants. Plant Biotechnol. Mol. Markers 18:60-77.

Sood, S. and P.K. Nagar. 2004. Changes in endogenous polyamines during flower development in two diverse species of rose. Plant Growth Regulat. 44:117-123.

Tarenghi, E. and J. Martin-Tanguy. 1995. Polyamines,floral induction and flower development of strawberry (Fragaria ananassa). Plant Growth Regulat. 17:157-165.

Wada, N., M. Shinozaki, and H. Iwamura. 1994. Flower induction by polyamines and related compounds in seedlings of morning glory (Pharbitis cv. Kidachi). Plant Cell Physiol. 35:469-472.

Wimalasekera, R., F. Tebartz, and G.F.E. Scherer. 2011. Polyamines, polyamine oxidases and nitric oxide in development, abiotic and biotic stresses. Plant Sci. 181:593-603.

Yang, Y.D., J.W. Su, L.W. Qu, W.D. Wang, N.N. Cui, and S.M. Guo. 2006. The preliminary study on the interaction relationship between flower bud differentiation in 'Jinba' and environmental factors. Liaoning Agr. Sci. 1:45-46.

Zhou, Q. and B.J. Yu. 2010. Changes in content of free, conjugated and bound polyamines and osmotic adjustment in adaptation of vetiver grass to water deficit. Plant Physiol Biochem. 48: 417-425. 